\title{
User Experiences with Nuclear Physics Calculations on a H2O Metacomputing System and on the BEgrid ${ }^{\star}$
}

\author{
P. Hellinckx, K. Vanmechelen, G. Stuer, F. Arickx, and J. Broeckhove \\ Department of Mathematics and Computer Science, \\ University of Antwerp, BE-2020 Antwerp, Belgium \\ peter.hellinckx@ua.ac.be
}

\begin{abstract}
We report on user experiences gathered with quantum nuclear scattering calculations on a $\mathrm{H} 2 \mathrm{O}$ based metacomputing system and on the BEgrid, the Belgian EGEE Grid infrastructure. We compare quantifiable aspects such as Grid utilization but also ease-of-use and the experience encountered in porting the application to each of the Grid environments.
\end{abstract}

\section{Introduction}

While Grids are becoming a fact of life in High Performance Computing (HPC) it is clear that the term stands for a whole range of middleware systems with strongly varying characteristics. Although traditional Grid systems often host an extensive stack of Grid services, many applications do not necessarily require explicit coordination and centralized services for authentication, registration, and resource brokering. For these applications, a lightweight model in which individuals and organizations share their superfluous resources on a peer-to-peer basis, seems to be more suitable [1].

In order to test this claim, we have implemented a quantum physics, nuclear 3-cluster scattering problem using both a lightweight and a heavyweight Grid. We chose this test case, of which the details will be described later, for several reasons. First, the computation has both a CPU-intensive and a data-intensive part. Secondly, the computation is too extensive to solve on a single machine, yet it does not require the full blown Grid infrastructure. Thirdly, this application fits an algorithmic approach that is rather common in many scientific computations.

This contribution presents the results of this comparative study. Our choice for a lightweight Grid system is $\mathrm{H} 2 \mathrm{O}$ [2], as we have considerable experience using this middleware. Our choice for a heavyweight Grid is the BEgrid which hosts the Belgian part of the EGEE project. As a member of the BEgrid consortium, our research group has experience in its procedures and maintenance,

\footnotetext{
* Research supported in part by the Ministerie van de Vlaamse Gemeenschap, Afdeling Technologie en Innovatie as part of the Vlaamse pilot-gridinfrastructuur project.
} 
as well as in gridifying applications. In what follows, the design, experimental results and a performance comparison between both platforms will be discussed.

\section{Problem Statement}

To obtain physical properties of quantum systems, such as atoms, nuclei or molecules, one needs to solve the so-called Schrödinger equation. In order to solve it, proper boundary conditions must be chosen. The solutions, the so-called "wave-functions" then allow for the calculation of physical quantities. The equation and its boundary conditions are usually too complex to be solved for manybody systems (e.g. a nucleus), and approximations have to be introduced. One approach is to expand the wave-function on a discrete, infinite-dimensional, set of basis states. Substitution of this approximation in the equation and boundary conditions, leads to a much simpler matrix equation in the expansion coefficients to be solved.

The matrix formulation can be further simplified by choosing expansion bases with specific properties. The Modified J-Matrix (MJM) model 3] is such an approach, and has been applied to 3-cluster nuclear systems [4. We consider it here to obtain scattering results for a 3-particle configuration of a triton and 2 neutrons of ${ }^{5} \mathrm{H}$. The calculations essentially consist of two steps: (1) a CPUintensive calculation of the matrix elements in the matrix equation, and (2) the solution of the matrix equation. Step 2 can be obtained in reasonable time on a single node, and we will therefore only consider the gridification of step 1 .

The oscillator expansion basis for the solution of a 3-cluster problem, is enumerated by a set of indices. These are the hypermoment $K$, describing the three cluster geometry; relative angular momenta $l_{1}$ and $l_{2}$ between the three clusters coupled to the total angular momentum $L$, a constant of motion; the oscillator index $n$. The number of $\left(l_{1} l_{2}\right)$ combinations depends on both $K$ and $L$. The essential matrix to be determined is the energy matrix, denoted by

$$
\left\langle K_{i},\left(l_{1} l_{2}\right)_{i} L, n_{i}|\hat{H}| K_{j},\left(l_{1} l_{2}\right)_{j} L, n_{j}\right\rangle=\left\langle K_{i}, l_{i}, n_{i}|\hat{H}| K_{j}, l_{j}, n_{j}\right\rangle
$$

where $\hat{H}$ is the Hamiltonian, or energy, operator, and $i$ and $j$ distinguish basis states; the right hand side in (11) simplifies the notation, omitting $L$ as an overall constant, and replacing the combination $\left(l_{1} l_{2}\right)_{i}$ by $l_{i}$.

The theory to obtain (11) 4] is well beyond the scope of this paper, but it can be broken down to

$$
\left\langle\left\langle K_{i}, l_{i}|\hat{H}| K_{j}, l_{j}\right\rangle\right\rangle=\sum_{t} \sum_{l_{r}} \sum_{l_{s}} R\left(K_{i}, l_{i}, l_{r}, t\right)\left\langle\left\langle K_{i}, l_{r}|\hat{H}| K_{j}, l_{s}\right\rangle\right\rangle_{t} R\left(K_{j}, l_{s}, l_{j}, t\right)
$$


where $\langle\langle\rangle\rangle$ stands for a matrix over all $n_{i}, n_{j}$ indices. The $R$ factors are socalled Raynal-Revai coefficients as discussed in [4, and the nature and range of index $t$ depends on the nucleus $\left({ }^{5} H\right)$ and its cluster decomposition $(t+n+n)$.

The granularity of the problem is clear from (2), and reduces the problem to a fork and join algorithm by calculating all independent $\left\langle\left\langle K_{i}, l_{r}|\hat{H}| K_{j}, l_{s}\right\rangle\right\rangle_{t}$ matrices for fixed $K_{i}, K_{j}$ and all allowed combinations $l_{r}, l_{s}, t$ (the fork), followed by a summation to obtain (21) (the join). In this paper we discuss a calculation for $L=0$ and $K=0,2,4, . ., 16$, a range of $l_{1}=l_{2}=0,1, . ., K / 2$ values, and $45 t$ values. All of the computational code components are implemented in Fortran90 using the Intel v7 compiler.

A farmer-worker distribution algorithmic model seems an evident choice for this problem, because all of the fork tasks are independent of one another. The tasks are file based, meaning that they get their input from a series of files and write their results into one. All input files except one, a configuration file which contains the particular indices for the current computation, have a constant content for all tasks.

\section{$3 \mathrm{H} 2 \mathrm{O}$}

$\mathrm{H} 2 \mathrm{O}[2]$ is developed by Emory University and is a component-based, serviceoriented framework for distributed metacomputing. $\mathrm{H} 2 \mathrm{O}$ is designed to cater for lightweight, general purpose, loosely coupled networks where resources are shared on a P2P basis. Adopting a provider-centric view of resource sharing, it provides a lightweight, general-purpose, Java based, configurable platform. $\mathrm{H} 2 \mathrm{O}$ adopts the microkernel design philosophy: resource owners host a software backplane (called a kernel) onto which owners, clients, or third-party resellers may load components (called pluglets) that deliver value added services. It is this last aspect that makes $\mathrm{H} 2 \mathrm{O}$ unique: by separating the service provider from the resource owner, it becomes possible for any authorized third party to deploy new services in a running kernel.

\subsection{Software Architecture}

Since $\mathrm{H} 2 \mathrm{O}$ basically stands for an empty service container, no infrastructural Grid components exist. In order to keep the $\mathrm{H} 2 \mathrm{O}$ approach as lightweight as possible, we implemented the infrastructural components which are absolutely necessary: a lookup service (LUS), a file transfer pluglet (FTPl), a broker, and executors. Figure 1 shows the basic architecture of our framework.

Before the calculation can start, the infrastructure has to be set up. This comes down to starting the LUS, and initiating the $\mathrm{H} 2 \mathrm{O}$ kernel on all participating nodes. These kernels will register themselves with the LUS and deploy the FTPl. From this point on, the system is ready to start the calculation. 


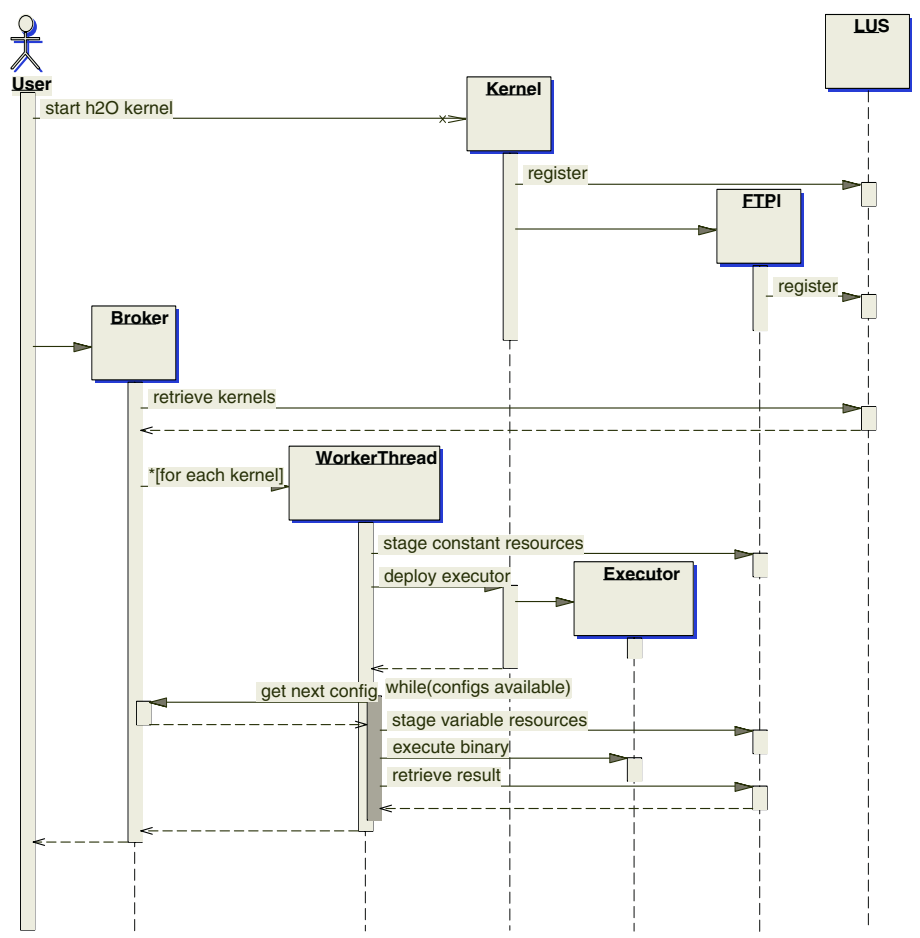

Fig. 1. Architecture of the framework

The first step in distributing the problem involves initializing the broker component which is responsible for the overall progress of the calculation. It will locate all available kernels using the LUS and instantiate WorkerThreads for all of them. Each one is responsible for the communication with one particular kernel. They start by staging the constant-content input files and the Fortran binary using the FTPl, followed by deploying the executor pluglet. Next, as long as there are sets of indices left to calculate, they will generate configuration files, transfer them to the kernel, start the executor, and retrieve the result. Finally, when all required combinations have been calculated, this process stops and the results can be recombined.

\section{BEgrid}

The BEgrid project involves an infrastructural collaboration between several universities and research institutions in Belgium. It is currently running LCG 2.2 middleware [5] and includes sites from the universities of Antwerp, Ghent, Leuven and Brussels as well as other research oriented institutions such as the Flanders Marine Institute. More institutions are expected to join on a short term 
basis. BEgrid is part of the North European ROC region of the EGEE project. Many sites are currently in deployment phase, 205 CPUs will be operational in the beginning of 2005 .

\subsection{Software Architecture}

The LCG middleware comprises of a number of functional modules that cooperate to provide workload, data and information management services. A resource broker matches job requirements, which are expressed in JDL (Job Description Language), to the available resources. The user has the ability to state complex infrastructural and operational job requirements using a standardized information schema called GLUE. The broker also supports user-defined ranking of resources that fulfill the hard job requirements, based on soft QoS requirements such as the average queued time for a job at a compute resource. After a computing resource is selected by the broker, the job is forwarded to the computing element (CE) associated with that resource. The computing element virtualizes compute resources such as PBS, LSF or Condor farms, providing uniform access to heterogeneous compute back-ends.

In the same way as the $\mathrm{CE}$ provides a virtualization layer for compute resources, a storage element (SE) delivers such a layer for heterogeneous storage resources. A replica management service (RMS) supports file based data replication and orchestrated access to files stored across SEs. Although jobs may interact with the RMS to access large data files, small I/O requirements can be fulfilled by specifying input and output sandboxes. These allow the user to indicate which files should be staged in and out before and after the computation. All interactions with the Grid infrastructure take place from a user interface node which supports APIs, command line, and graphical interfaces to BEgrid.

The LCG middleware supports a batch oriented computing model, and is currently in process of providing better support for data dependencies between jobs and more tightly coupled computations. This does not affect the mapping of our problem to the LCG based computing infrastructure as jobs are trivially parallel. A characteristic that does affect our job distribution scheme is the fact that long delays have been observed when submitting jobs and receiving their results on a zero loaded computing resource. The computation time for individual indices also varies widely from a few seconds to a few hours. Therefore it was deemed necessary to group multiple calculations in a single job, in order to mitigate the performance penalty induced by these delays. To determine the size of these groupings we monitored the number of queued jobs over all $\mathrm{CE}$ queues during the computation and adjusted the group size accordingly. The idea is that as long as all CE queues are sufficiently loaded to keep the compute resources busy, the submission delay will not affect the total wall-clock time of the computation.

All file staging was performed using the input and output sandboxes since the calculations are compute intensive. After jobs finish, a polling component on the user interface fetches the job output. 


\section{$5 \quad$ Experimental Results}

\section{$5.1 \quad$ Speedup}

The distributed computations on the $\mathrm{H} 2 \mathrm{O}$ framework were performed on a local cluster containing a heterogeneous set of 20 PC-nodes with an Intel processor ranging from 1.7 to $2.66 \mathrm{GHz}$, part of a $100 \mathrm{Mb}$ LAN. For the BEgrid tests, we used $243.2 \mathrm{GHz}$ Pentium 4 nodes at Antwerp and 8 dual-Opteron/244 nodes (1.8 $\mathrm{GHz}$ ) at Leuven. This amounts to 33 normalized $3.2 \mathrm{CPUs}$ which is the maximum reachable speedup factor on this setup. Both sites use 1 GigE interconnect. The user interface and resource broker were both located at Antwerp.

Table 1 shows the turn-around time of the distributed computations for different values of $K$, and compares these to the pure sequential execution timing. The speedup factor which denotes the ratio between sequential and distributed execution time is also shown for each $\mathrm{K}$. The table depicts low speedup figures for computations with $K<10$ both for $\mathrm{H} 2 \mathrm{O}$ and BEgrid. More time is spent deploying the jobs than processing them. The reason is that most of the tasks with low $K$ have a very small execution time. From $K=10$ onwards, the picture changes, as the mean execution time for these jobs rises significantly. The results are almost optimal for $K>12$ on $\mathrm{H} 2 \mathrm{O}$. For $\mathrm{H} 2 \mathrm{O}$, the low speedup for small $K$ is a direct consequence of our naive implementation of the Grid infrastructure components. Prefetching the data files will probably boost speedup for small $K$.

For BEgrid, low speedup values can be attributed to job submission delays which are in the order of seconds, and result fetching delays which are in the order of minutes. These make it difficult to achieve optimal resource utilization in the beginning and at the end of the computation. The delays were not found to be caused by data transmission overhead but rather by the workload management system itself.

Table 1. Speedup achieved on the available H2O and LCG resources

\begin{tabular}{|r|rrr|rrr|}
\hline & \multicolumn{3}{|c|}{ H2O } & \multicolumn{3}{c|}{ LCG } \\
\hline $\mathrm{K}$ & sequential distributed speedup & \multicolumn{3}{|c|}{ sequential distributed speedup } \\
\hline 0 & $2 \mathrm{~s}$ & $55 \mathrm{~s}$ & 0.04 & $1 \mathrm{~s}$ & $7 \mathrm{~m} 57 \mathrm{~s}$ & 0.00 \\
2 & $9 \mathrm{~s}$ & $1 \mathrm{~m} 26 \mathrm{~s}$ & 0.11 & $3 \mathrm{~s}$ & $9 \mathrm{~m} 00 \mathrm{~s}$ & 0.01 \\
4 & $1 \mathrm{~m} 11 \mathrm{~s}$ & $2 \mathrm{~m} 24 \mathrm{~s}$ & 0.49 & $35 \mathrm{~s}$ & $16 \mathrm{~m} 27 \mathrm{~s}$ & 0.04 \\
6 & $9 \mathrm{~m} 01 \mathrm{~s}$ & $4 \mathrm{~m} 00 \mathrm{~s}$ & 2.25 & $5 \mathrm{~m} 19 \mathrm{~s}$ & $16 \mathrm{~m} 59 \mathrm{~s}$ & 0.32 \\
8 & $56 \mathrm{~m} 31 \mathrm{~s}$ & $8 \mathrm{~m} 25 \mathrm{~s}$ & 6.72 & $30 \mathrm{~m} 44 \mathrm{~s}$ & $18 \mathrm{~m} 54 \mathrm{~s}$ & 1.63 \\
10 & $4 \mathrm{~h} 52 \mathrm{~m} 56 \mathrm{~s}$ & $20 \mathrm{~m} 22 \mathrm{~s}$ & 14.38 & $2 \mathrm{~h} 55 \mathrm{~m} 43 \mathrm{~s}$ & $18 \mathrm{~m} 42 \mathrm{~s}$ & 9.40 \\
12 & $21 \mathrm{~h} 33 \mathrm{~m} 12 \mathrm{~s}$ & $1 \mathrm{~h} 12 \mathrm{~m} 52 \mathrm{~s}$ & 17.75 & $12 \mathrm{~h} 37 \mathrm{~m} 12 \mathrm{~s}$ & $49 \mathrm{~m} 36 \mathrm{~s}$ & 15.27 \\
14 & $82 \mathrm{~h} 38 \mathrm{~m} 21 \mathrm{~s}$ & $4 \mathrm{~h} 11 \mathrm{~m} 24 \mathrm{~s}$ & 19.72 & $49 \mathrm{~h} 39 \mathrm{~m} 20 \mathrm{~s}$ & $2 \mathrm{~h} 01 \mathrm{~m} 05 \mathrm{~s}$ & 24.61 \\
16 & $285 \mathrm{~h} 04 \mathrm{~m} 20 \mathrm{~s} 14 \mathrm{~h} 23 \mathrm{~m} 59 \mathrm{~s}$ & 19.80 & $173 \mathrm{~h} 37 \mathrm{~m} 26 \mathrm{~s}$ & $6 \mathrm{~h} 07 \mathrm{~m} 31 \mathrm{~s}$ & 28.30 \\
\hline
\end{tabular}

\section{$5.2 \quad$ Utilization}

Turn-around timing is not an acceptable measure if we wish to compare distributive platforms, such as the $\mathrm{H} 2 \mathrm{O}$ and LCG middlewares, as the amount of 
available resources varies significantly from one run to another. Therefore, we consider the utilization rate of the participating nodes, which is defined as the percentage of time the processor is performing the actual computation. This measure provides an indication of the overhead introduced by both the middleware and data transfers, and is largely independent of the hardware used. Figure 2 shows the utilization as a function of $K$, which indicates strongly decreasing overhead for increasing $K$-values. For $\mathrm{H} 2 \mathrm{O}$, the main part of the overhead can be attributed to data transfers. The remaining part, $0.03 \%$ to $0.07 \%$ of the total turn-around time, is middleware overhead. For BEgrid, job submission and result fetching delays were the main causes for overhead.

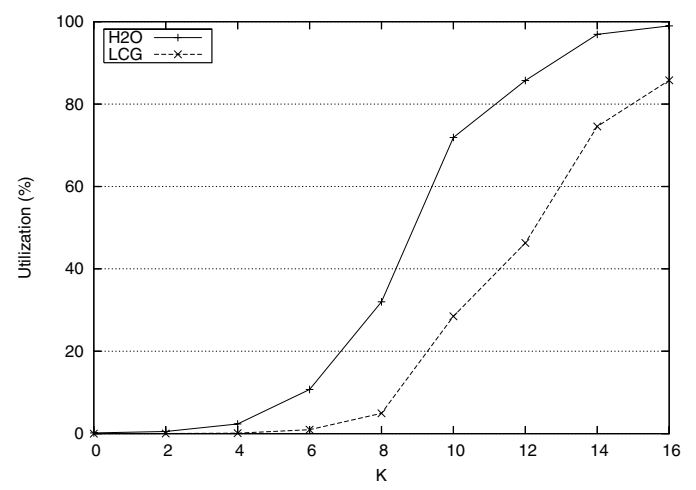

Fig. 2. Processor Utilization Comparison between $\mathrm{H} 2 \mathrm{O}$ and BEgrid

\section{$6 \mathrm{H} 2 \mathrm{O}$ vs. BEgrid}

The main merits of $\mathrm{H} 2 \mathrm{O}$ are that it is lightweight and easy to deploy. As it is Java based, it functions on any desktop commodity PC available, limiting hardware investments. Furthermore, job submission is very responsive, which allows for the submission of small jobs, without creating too much overhead. Finally, its component based architecture allows for on-demand deployment of custom made, and highly optimized Grid components such as specialized schedulers, LUS, etc.

The latter advantage also turns out to be a shortcoming since every component has to be constructed from scratch. As the available documentation is rather scarce, this results in an initially steep learning curve. In the current implementation, we opted for a minimalistic approach which kept the infrastructural components simple, but unfortunately also unscalable and, in general, not reusable. High quality, scalable, robust, and general purpose components would require an important implementation investment. Finally, though H2O may be written in Java, typical computational components are not. Thus special provisions have to be made to ensure that kernels hosted on Linux, Solaris, Mac and Windows platforms can participate in the computation. 
One of the main merits of distributing the computation on BEgrid is the efficiency of the implementation process itself: only 230 lines of bash scripting code were needed to capture all job distribution and execution logic. Though the highly parallel and independent nature of the distributed subtasks is certainly of influence, this is also directly related to the fact that core Grid services for workload, data and information management as well as core security provisions, are delivered 'out-of-the-box'. Another important advantage is the fact that coding an application to a model and infrastructure accepted on a European scale, opens the possibility for accessing a large scale infrastructure. On the other hand, one should be aware that gaining access to a general purpose (inter)national Grid resources still requires "social" interactions for the application to be accepted at remote sites.

A potential shortcoming of BEgrid is the high job submission and result fetching delay which may affect applications that have to face a fine grained level of job distribution. Although our approach of wrapping multiple application level jobs into a single Grid job mitigated these effects, it was still difficult to achieve a good load balance at the beginning and near the end of the total computation.

\section{Conclusions}

The main conclusion of this work is that both Grid platforms are well suited for gridifying a highly parallelizable CPU-intensive application.

In its default configuration, $\mathrm{H} 2 \mathrm{O}$ is best suited for distributive computations on a limited scale. It is highly responsive, but only provides the base Javaoriented infrastructure to develop Grid applications. It does allow for a more scalable approach, but requires all necessary Grid components for this to be custom made. In order to overcome this drawback, a pluggable $\mathrm{H} 2 \mathrm{O}$ based Grid infrastructure is currently under development.

BEgrid provides a platform with standardized well-defined services for distributive processing. This delimited set of services to approach the Grid is a merit of the system, because of its straightforward usage, as well as a shortcoming because of its inherent limitations. With respect to computing resources, it features international scalability, which is an important asset for large scale distributed applications.

\section{References}

1. Jonathan Chin, Peter V. Coveney: Towards tractable toolkits for the Grid: a plea for lightweight, usable middleware. http://www.realitygrid.org/lgpaper.html

2. V. Sunderam, D. Kurzyniec: Lightweight Self-Organizing Frameworks for Metacomputing. In 11th Int. Symp. on High Performance Distributed Computing, 2002. 
3. J. Broeckhove, F. Arickx, W. Vanroose and V. Vasilevsky: The Modified J-Matrix method for Short-Range Potentials. J. Phys. A: Math. Gen 37 (2004) 1-13

4. V. S. Vasilevsky, A. V. Nesterov, F. Arickx and J. Broeckhove: The Algebraic Model for Scattering in Three-s-cluster Systems: Theoretical Background. Phys. Rev. C63 (2001) 034606:1-16

5. A. D. Delgado et al., LCG-2 User Guide. https://edms.cern.ch/file/454439/LCG2-Userguide.pdf. 